\title{
Direct correlation functions of the Widom-Rowlinson model
}

\author{
R. Fantoni* and G. Pastore ${ }^{\dagger}$ \\ Dipartimento di Fisica Teorica dell' Università and Istituto Nazionale di Fisica della Materia, \\ Strada Costiera 11, 34014 Trieste, Italy
}

(Dated: October 3, 2018)

\begin{abstract}
We calculate, through Monte Carlo numerical simulations, the partial total and direct correlation functions of the three dimensional symmetric Widom-Rowlinson mixture. We find that the differences between the partial direct correlation functions from simulation and from the Percus-Yevick approximation (calculated analytically by Ahn and Lebowitz) are well fitted by Gaussians. We provide an analytical expression for the fit parameters as function of the density. We also present Monte Carlo simulation data for the direct correlation functions of a couple of non additive hard sphere systems to discuss the modification induced by finite like diameters.
\end{abstract}

PACS numbers: 61.20.Ja,61.20.Gy

KEYWORDS: Widom-Rowlinson model, direct correlation function.

\section{INTRODUCTION}

Fluid binary mixtures may exhibit the phenomenon of phase separation. The simplest system able to undergo a demixing phase transition is the model introduced by Widom and Rowlinson some years ago [1]. Consider a binary mixture of non-additive hard spheres. This is a fluid made of hard spheres of specie 1 of diameter $R_{11}$ and number density $\rho_{1}$ and hard spheres of specie 2 of diameter $R_{22}$ and number density $\rho_{2}$, with a pair interaction potential

\footnotetext{
* Address: Dipartimento di Fisica Teorica dell' Universita' degli Studi di Trieste, Strada Costiera 11, 34014 Trieste, Italy; Phone: +39 040 2240608; Fax: +39 040 224601; Electronic address: rfantoni@ts.infn.it

† Address: Dipartimento di Fisica Teorica dell' Universita' degli Studi di Trieste, Strada Costiera 11, 34014 Trieste, Italy; Phone: +39 040 2240242; Fax: +39 040 224601; Electronic address: pastore@ts.infn.it
} 
between species $i$ and $j$ that can be written as follows

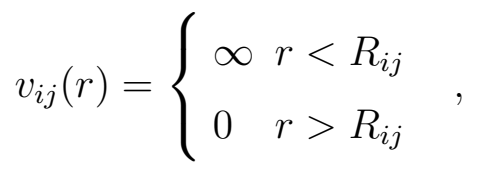

where $R_{12}=\left(R_{11}+R_{22}\right) / 2+\alpha$. The Widom-Rowlinson (WR) model is obtained choosing the diameters of the spheres equal to 0 ,

$$
R_{11}=R_{22}=0
$$

so that there is no interaction between like spheres and there is a hard core repulsion of diameter $\alpha$ between unlike spheres. The symmetry of the system induces the symmetry of the unlike correlations $\left[h_{12}(r)=h_{21}(r), \quad c_{12}(r)=c_{21}(r)\right]$. The WR model has been studied in the past by exact [2] and approximate [3, 4, 5], 6] methods and it has been shown that it exhibits a phase transition at high density. More recently, additional studies have appeared and theoretical predictions have been confirmed by Monte Carlo (MC) computer simulations [7, 8, 9, 10]

In this paper we will study the three dimensional symmetric Widom-Rowlinson mixture for which $\rho_{1}=\rho_{2}=\rho / 2$, where $\rho$ is the total number density of the fluid, and

$$
\begin{aligned}
& h_{11}(r)=h_{22}(r), \\
& c_{11}(r)=c_{22}(r) .
\end{aligned}
$$

Moreover we know from (II) that the partial pair correlation function $g_{i j}=h_{i j}+1$ must obey

$$
g_{i j}(r)=0 \quad \text { for } r<R_{i j}
$$

Our main goal is to focus on the direct correlation functions (dcf) of the WR model as a simplified prototype of non-additive hard spheres (NAHS) systems. The reasons to focus on the dcf's is twofold: on the one hand, they are easier functions to model and fit. On the other hand, they play a central role in approximate theories like the Percus-Yevick approximation or mean spherical approximation (MSA) 11]. We hope that a better understanding of the dcf's properties in the WR model, could help in developing accurate analytical theories for the NAHS systems.

We calculate through Monte Carlo simulations the like $g_{11}^{(M C)}(r)$ and unlike $g_{12}^{(M C)}(r)$ pair distribution functions for a system large enough to allow a meaningful determination of the 
correspondent partial direct correlation functions $c_{11}^{(M C)}(r)$ and $c_{12}^{(M C)}(r)$, using the OrnsteinZernike equation [11]. We compare the results for the unlike direct correlation function with the results of the Percus-Yevick (PY) analytic solution found by Ahn and Lebowitz [3, 4]. In the same spirit as the work of Grundke and Henderson for a mixture of additive hard spheres 12], we propose a fit for the functions $\Delta_{11}^{c}(r)=c_{11}^{(M C)}(r)$ and $\Delta_{12}^{c}(r)=c_{12}^{(M C)}(r)-c_{12}^{(P Y)}(r)$.

At the end of the paper we also show the results from two Monte Carlo simulations on a mixture of non-additive hard spheres with equal diameter spheres $R_{11}=R_{22}=R_{12} / 2$ and on one with different diameter spheres $R_{11}=0$ and $R_{22}=R_{12}$ to study the effect of non zero like diameters on the WR dcf's.

\section{MONTE CARLO SIMULATION AND PY SOLUTION}

The Monte Carlo simulation was performed with a standard NVT Metropolis algorithm 13] using $N=4000$ particles. Linked lists [13] have been used to reduce the computational cost. We generally used $5.2 \times 10^{8}$ Monte Carlo steps where one step corresponds to the attempt to move a single particle. The typical CPU time for each density was around 20 hours (runs at higher densities took longer than runs at smaller densities) on a Compaq AlphaServer 4100 5/533.

We run the simulation of WR model at 6 different densities $\bar{\rho}=\rho \alpha^{3}=0.28748,0.4,0.45$, $0.5,0.575$, and 0.65 . Notice that the most recent computer simulation calculations $[9,10]$ give consistent estimates of the critical density around 0.75 . Our data at the highest density (0.65) are consistent with a one phase system.

The Monte Carlo simulation returned the $g_{i j}(r)$ over a range not less than $9.175 \alpha$ for the densest system. In all the studied cases the pair distribution functions attained their asymptotic value well inside the maximum distance they were evaluated. Thus, it has been possible to obtain accurate fourier transforms of the correlation functions $\left[h_{i j}(k)\right]$. To obtain the $c_{i j}(r)$ we used Ornstein-Zernike equation as follows

$$
\begin{aligned}
& c_{11}(k)=\frac{h_{11}(k)\left[1+\frac{\rho}{2} h_{11}(k)\right]-\frac{\rho}{2} h_{12}^{2}(k)}{\left[1+\frac{\rho}{2} h_{11}(k)\right]^{2}-\left[\frac{\rho}{2} h_{12}(k)\right]^{2}} \\
& c_{12}(k)=\frac{h_{12}(k)}{\left[1+\frac{\rho}{2} h_{11}(k)\right]^{2}-\left[\frac{\rho}{2} h_{12}(k)\right]^{2}}
\end{aligned}
$$

From the $h_{i j}(k)$ and $c_{i j}(k)$ we get the difference $\gamma_{i j}(k)=h_{i j}(k)-c_{i j}(k)$ which is the fourier 
transform of a continuous function in real space. So it is safe to transform back in real space [to get $\gamma_{i j}(r)$ ]. Finally, the dcf's are obtained from the differences $h_{i j}(r)-\gamma_{i j}(r)$.

While for a system of non-additive hard sphere in three dimensions a closed form solution to the PY approximation is still lacking, Ahn and Lebowitz have found an analytic solution of this approximation for the WR model (in one and three dimensions).

The PY approximation consists of the assumption that $c_{i j}(r)$ does not extend beyond the range of the potential

$$
c_{i j}(r)=0 \quad \text { for } r>R_{i j}
$$

Combining this with the exact relation (15) and using the Ornstein-Zernike equation we are left with a set of equations for $c_{i j}(r)$ and $g_{i j}(r)$ which have been solved analytically by Ahn and Lebowitz.

Their solution is parameterized by a parameter $z_{0}$. They introduce the following two functions of $z_{0}$ (which can be written in terms of elliptic integrals of the first and third kind)

$$
\begin{aligned}
& I_{1} \equiv \int_{z_{0}}^{\infty} \frac{d z}{z \sqrt{z^{3}+4 z / z_{0}-4}} \\
& I_{2} \equiv \int_{z_{0}}^{\infty} \frac{d z}{\sqrt{z^{3}+4 z / z_{0}-4}}
\end{aligned}
$$

and define $z_{0}$ in terms of the partial densities $\rho_{1}$ and $\rho_{2}$ as follows

$$
\eta \equiv 2 \pi \sqrt{\rho_{1} \rho_{2}}=\frac{\left(I_{2} / 2\right)^{3}}{\cos I_{1}} .
$$

They then define the following functions (note that in the last equality of equation (3.76) in 4] there is a misprint)

$$
\begin{aligned}
\bar{c}_{12}(k) \equiv & -\frac{2}{\sqrt{\rho_{1} \rho_{2}}} \sqrt{\frac{1+Y}{z_{0}^{3} Y^{3}+4 Y+4}} \\
& \times \sin \left[\frac{1}{2} \sqrt{z_{0}^{3} Y^{3}+4 Y+4} \int_{1}^{\infty} \frac{d z}{(z+Y) \sqrt{z_{0}^{3} z^{3}+4 z-4}}\right], \\
\bar{h}_{12}(k) \equiv & \bar{c}_{12}(k)\left[1-\rho_{1} \rho_{2} \bar{c}_{12}(k)\right],
\end{aligned}
$$

where $Y \equiv\left(2 k / I_{2}\right)^{2}$.

We also realized that some other misprint should be present in the Ahn and Lebowitz paper since we have found empirically that the PY solution (with $k$ in units of $\alpha$ ) should be given by

$$
c_{12}(k)=\bar{c}_{12}(k s),
$$


where $s$ is a scale parameter to be determined as follows

$$
s=-\left[\bar{h}_{12}(r=0)\right]^{1 / 3}
$$

Notice that for the symmetric case $\rho_{1}=\rho_{2}=\rho / 2$ and $\eta=\pi \rho=0.90316 \ldots$ we find $z_{0}=1$ and $s=1$.

In Figs. 1, 2, and 3 we show three cases corresponding to the extreme and one intermediate density. In the figures, we compare the MC simulation data with the PY solution for the partial pair distribution functions and the partial direct correlation functions. Our results for the partial pair distribution functions at $\rho \alpha^{3}=0.65$ are in good agreement with the ones of Shew and Yethiraj [9]. The figures show how the like correlation functions obtained from the PY approximation are the ones that differ most from the MC simulation data. The difference is more marked in a neighborhood of $r=0$ and becomes more pronounced as the density increases.

\section{FIT OF THE DATA}

From the simulations we found that $c_{12}^{(M C)}(r)<8 \times 10^{-3}$ for $r>\alpha$ at all the densities studied. This allows us to say that $\Delta_{12}^{c}(r) \simeq 0$ for $r>\alpha$. Moreover we found that both $\Delta_{12}^{c}(r)$ for $r<\alpha$, and $\Delta_{11}^{c}(r)$ are very well fitted by Gaussians

$$
\begin{aligned}
& \Delta_{11}^{c}(r) \simeq b_{11} \exp \left[-a_{11}\left(r+d_{11}\right)^{2}\right] \quad \text { for all } r>0, \\
& \Delta_{12}^{c}(r) \simeq b_{12} \exp \left[-a_{12} r^{2}\right] \quad \text { for } 0<r<\alpha,
\end{aligned}
$$

In Figs. 4 and $[5$ we show the behaviors of the parameters of the best fit (16) and (17), with density. In order to check the quality of the fit, we did not use the data at $\bar{\rho}=0.45$ in the determination of the parameters. The points for $a_{12}$ and $b_{12}$ are well fitted by a straight line or a parabola. As shown in Fig. 团 the best parabolae are

$$
\begin{aligned}
& a_{12}(\bar{\rho})=0.839+0.096 \bar{\rho}-1.287 \bar{\rho}^{2}, \\
& b_{12}(\bar{\rho})=-0.155+0.759 \bar{\rho}-0.159 \bar{\rho}^{2} .
\end{aligned}
$$


Fig. 5 shows how the parameters for $\Delta_{11}^{c}(r)$ are much more scattered and hard to fit. The quartic polynomial going through the five points, for each coefficient, are

$$
\begin{aligned}
& a_{11}(\bar{\rho})=-55.25+504 \cdot 8 \bar{\rho}-1659 \cdot \bar{\rho}^{2}+2364 \cdot \bar{\rho}^{3}-1236 \cdot \bar{\rho}^{4} \\
& b_{11}(\bar{\rho})=171.4-1556 \cdot \bar{\rho}+5166 \cdot \bar{\rho}^{2}-7421 \cdot \bar{\rho}^{3}+3906 \cdot \bar{\rho}^{4} \\
& d_{11}(\bar{\rho})=128.9-1144 \cdot \bar{\rho}+3747 \cdot \bar{\rho}^{2}-5328 \cdot \bar{\rho}^{3}+2782 \cdot \bar{\rho}^{4}
\end{aligned}
$$

The difficulty in finding a good fit for these parameters may be twofold: first we are fitting $\Delta_{11}^{c}(r)$ with a three (instead of two) parameters curve and second the partial pair distribution functions obtained from the Monte Carlo simulation are less accurate in a neighborhood of the origin (due to the reduced statistics there). This inaccuracy is amplified in the process of finding the partial direct correlation functions. Such inaccuracy will not affect significantly $\Delta_{12}^{c}(r)$ which has a derivative very close or equal to zero near the origin, but it will significantly affect $\Delta_{11}^{c}(r)$ which is very steep near the origin.

In order to estimate the quality of the fit we have used the simulation data at $\bar{\rho}=0.45$. From Fig. 4 we can see how the parabolic fit is a very good one. In Fig. 5 the point at $\bar{\rho}=0.45$ gives an indication of the accuracy of the quartic fit. We have also compared the pair distribution and direct correlation functions obtained from the fit with those from MC: both the like and unlike distribution functions are well reproduced while there is a visible discrepancy in the dcf from the origin up to $r=0.5 \alpha$. However we expect that moving on the high density or low density regions (where the quartic polynomial becomes more steep) the quality of the fit will get worst. In particular the predicted negative values for $a_{11}$, in those regions, are completely unphysical and the fit should not be used to extrapolate beyond the range $0.28<\bar{\rho}<0.65$.

\section{FROM WR TO NON ADDITIVE HARD SPHERES}

In order to see how the structure, and in particular the dcf's of the Widom-Rowlinson model change as one switches on the spheres diameters we have made two additional Monte Carlo simulations. In the first one we chose $\rho_{1}=\rho_{2}=0.65 / R_{12}^{3}$ and $R_{11}=R_{22}=R_{12} / 2$. The resulting partial pair distribution functions and partial direct correlation functions are

shown in Fig. 6. From a comparison with Fig. 3 we see how in this case the switching on of the like diameters causes both $c_{12}(r)$ for $r<R_{12}$ and $g_{12}(r)$ for $r>R_{12}$ to approach $r=R_{12}$ 
with a slope close to zero.

In the second simulation we chose $\rho_{1}=\rho_{2}=0.65 / R_{12}^{3}$ and $R_{11}=0, R_{22}=R_{12}$. The resulting partial pair distribution functions and partial direct correlation functions are shown in Fig. 7. From a comparison with Fig. [3 we see how in this case the switching on of the like diameters causes both $g_{11}(0)$ and $c_{11}(0)$ to increase, and $c_{12}(r)$ to lose the nearly zero slope at $r=0$. As in the previous case $g_{12}(r)$ for $r>R_{12}$ approaches $r=R_{12}$ with a slope close to zero. The like 22 correlation functions for $r>R_{12}$ vary over a range comparable to the one over which vary the like 11 correlation functions of the WR model.

For both these cases there is no analytic solution of the PY approximation available and a better understanding of the behavior of the direct correlation functions may help in finding approximate expressions [14].

\section{CONCLUSIONS}

In this paper we have evaluated the direct correlation functions of a Widom-Rowlinson mixture at different densities through Monte Carlo simulation and we have studied the possibility of fitting the difference between MC data and the PY dcf's. We found a very good parameterization of $c_{12}(r)$ for $r<\alpha$ [see equations (17) and (18)-(19)] and a poorer one for $c_{11}(r)$ [see equations (16) and (20)-(22)]. The difficulty in this last case probably arises from the necessity of using three parameters [instead of just two needed for parameterizing $\left.c_{12}(r)\right]$, although it cannot be completely excluded some effect of the decreasing precision of the simulation data near the origin.

In the last part of the paper we have illustrated with additional Monte Carlo data the changes induced in the WR dcf's by a finite size of the excluded volume of like correlations. These results are meant to provide a guide in the search of a manageable, simple analytical parameterization of the structure of mixtures of non additive hard spheres which is still not available although highly desirable.

[1] B. Widom and J. Rowlinson, J. Chem. Phys. 15, 1670 (1970).

[2] D. Ruelle, Phys. Rev. Lett. 16, 1040 (1971).

[3] S. Ahn and J. L. Lebowitz, Phys. Lett. 44A, 424 (1973). 
[4] S. Ahn and J. L. Lebowitz, J. Chem. Phys. 60, 523 (1974).

[5] E. Bergmann, Mol. Phys. 32, 237 (1976).

[6] J. Karkheck and G. Stell, J. Chem. Phys. 71, 3620 (1979).

[7] H. Luo, C. Hoheisel, and J. Karkheck, Phys. Rev. A 42, 4690 (1976).

[8] R. Dickman and G. Stell, J. Chem. Phys. 102, 8674 (1995).

[9] C.-Y. Shew and A. Yethiraj, J. Chem. Phys. 104, 7665 (1996).

[10] G. Johnson, H. Gould, J. Machta, and L. K. Chayes, Phys. Rev. Lett. 79, 2612 (1997).

[11] J. P. Hansen and I. R. McDonald, "Theory of simple liquids" (Academic Press, London, 1986), 2nd ed.

[12] E. W. Grundke and D. Henderson, Molec. Phys. 24, 269 (1972).

[13] M. P. Allen and F. J. Tildesley, "Computer simulation of Liquids" (Clarendon Press, Oxford, 1987).

[14] D. Gazzillo and G. Pastore, Chem. Phys. Lett. 159, 388 (1989). 


\section{LIST OF FIGURES}

Fig. 1] Top panel: partial direct correlation functions obtained from the Monte Carlo simulation (points) with the $c_{12}^{(P Y)}(r)$ obtained from the PY approximation (line) at a density $\rho \alpha^{3}=0.28748$. Bottom panel: partial pair distribution functions obtained from the Monte Carlo simulation compared with the ones obtained from the PY approximation at the same density. The open circles and the dashed line: the like correlation functions. Closed circles and the continuous line: the unlike correlation functions.

Fig. 2 Same as in Fig. 1 at a density $\rho \alpha^{3}=0.4$.

Fig. 3 Same as in Fig.1 at a density $\rho \alpha^{3}=0.65$.

Fig. 4 We plot, for five different values of the density, the parameters $a_{12}$ (diagonal crosses) and $b_{12}$ (starred crosses) of the best Gaussian fit (17) to $\Delta_{12}^{c}(r)$ for $r<\alpha$, and fit them with parabolae (lines). The parameters at $\rho \alpha^{3}=0.45$ where not used for the parabolic fit and give an indication of the quality of the fit.

Fig. 5 We plot, for five different values of the density, the parameters $a_{11}, b_{11}$ and $d_{11}$ (stars) of the best Gaussian fit (16) to $\Delta_{11}^{c}(r)$, and draw the quartic polynomial (lines) through them. The parameters at $\rho \alpha^{3}=0.45$ where not used to determine the quartic polynomial and give an indication of the quality of the fit.

Fig. [6] Monte Carlo results at a density $\rho=\rho_{1}=\rho_{2}=0.65 / R_{12}^{3}$ for the partial direct correlation function (on top) and the partial pair distribution function (below) of a mixture of non additive hard spheres with $R_{11}=R_{22}=R_{12} / 2$. The open circles denote the like correlation functions. The closed circles denote the unlike correlation functions.

Fig. 0 Monte Carlo results at a density $\rho=\rho_{1}=\rho_{2}=0.65 / R_{12}^{3}$ for the partial direct correlation function (on top) and the partial pair distribution function (below) of a mixture of non additive hard spheres with $R_{11}=0$ and $R_{22}=R_{12}$. The open circles denote the like 11 correlation functions. The open triangles denote the like 22 correlation functions. The closed circles denote the unlike correlation functions. 


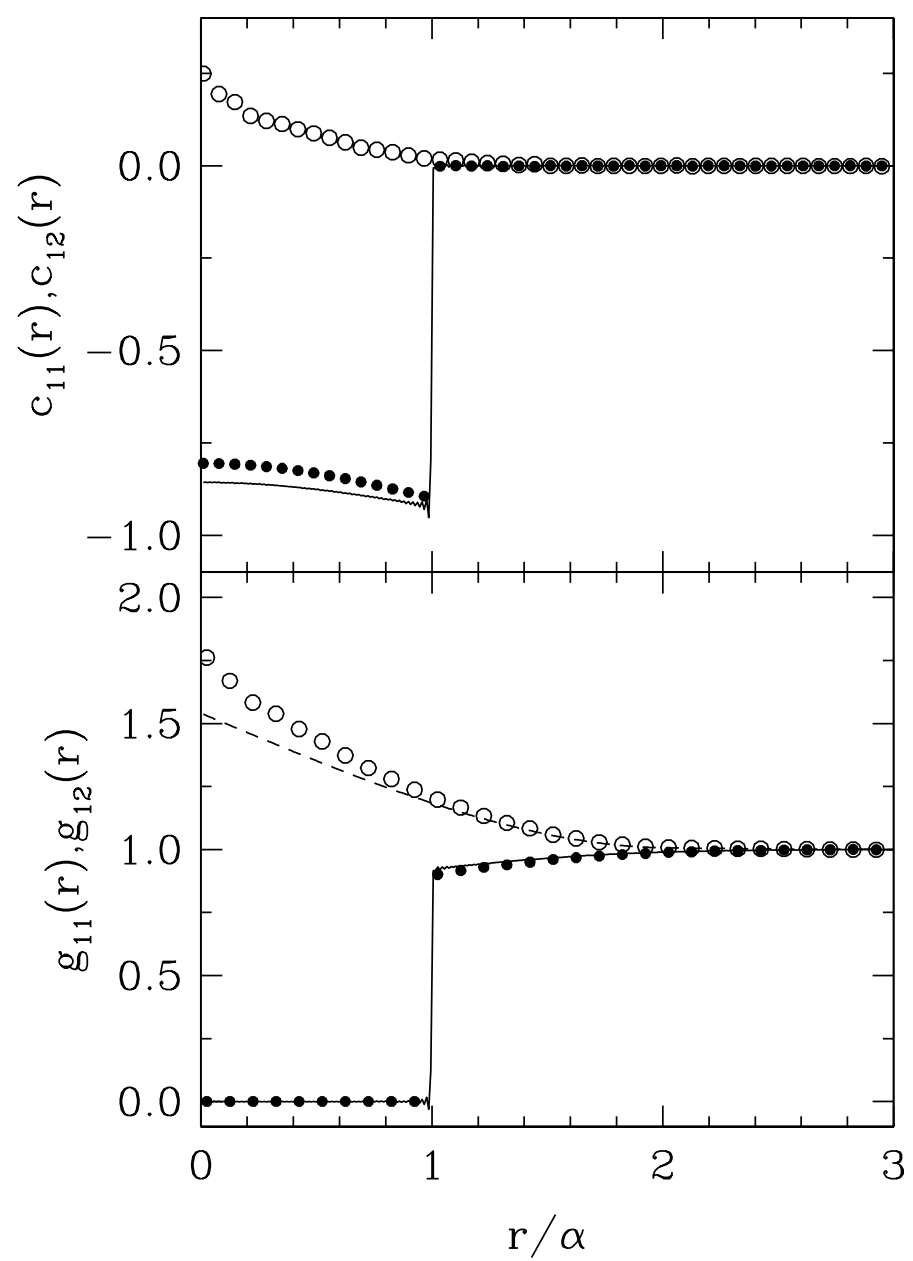

FIG. 1: R. Fantoni and G. Pastore 


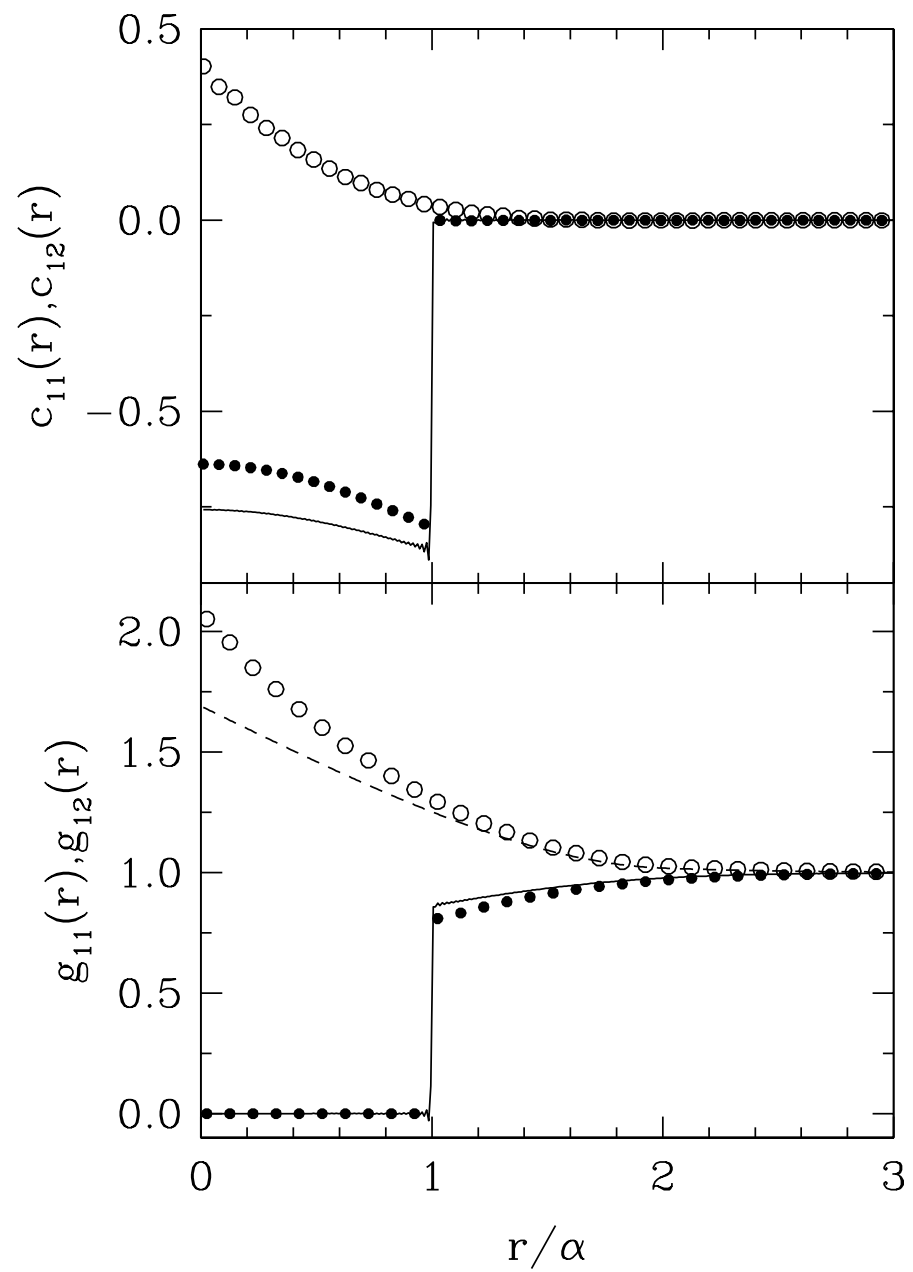

FIG. 2: R. Fantoni and G. Pastore 


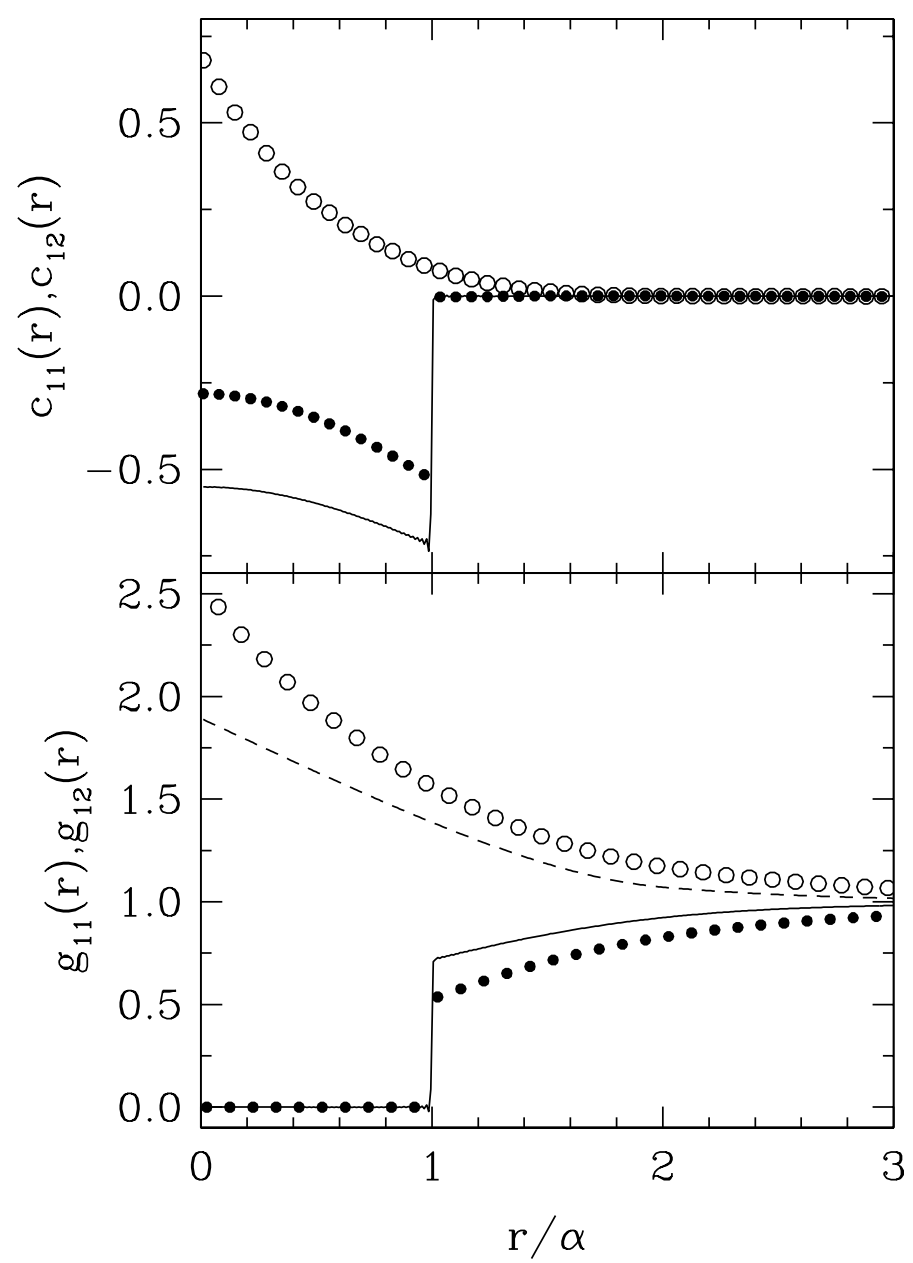

FIG. 3: R. Fantoni and G. Pastore 


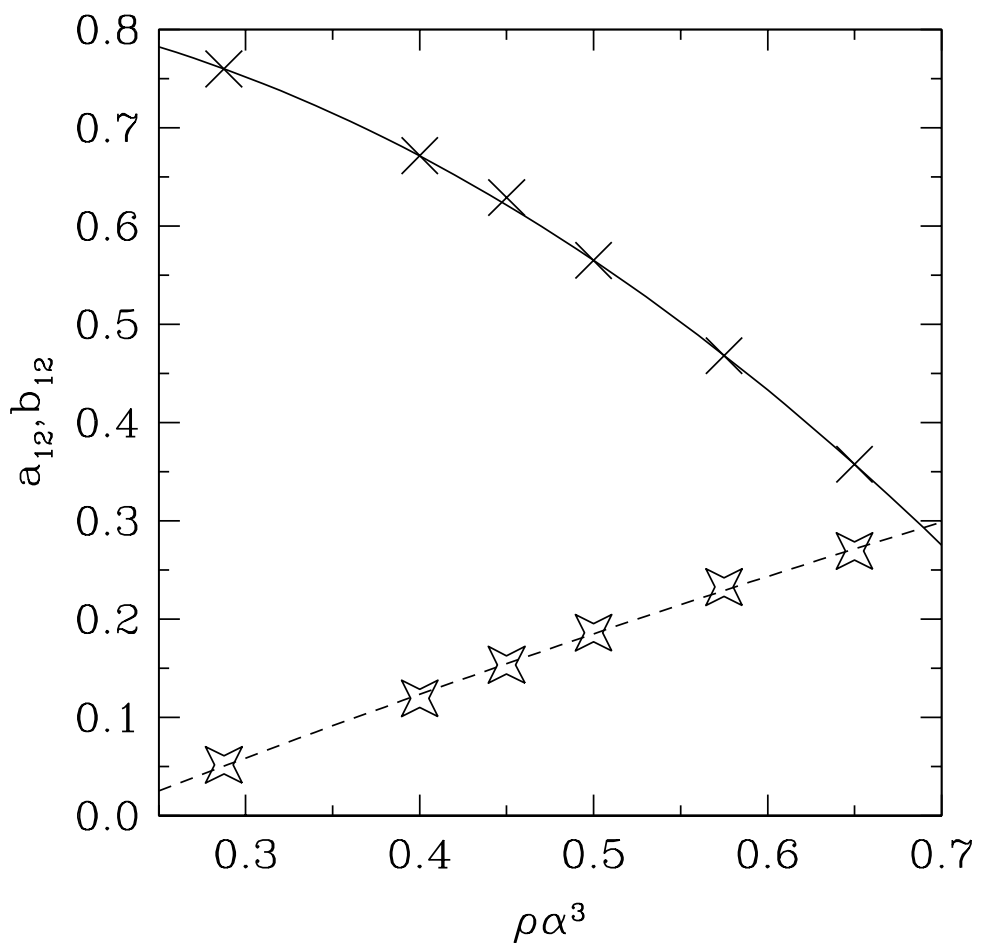

FIG. 4: R. Fantoni and G. Pastore 


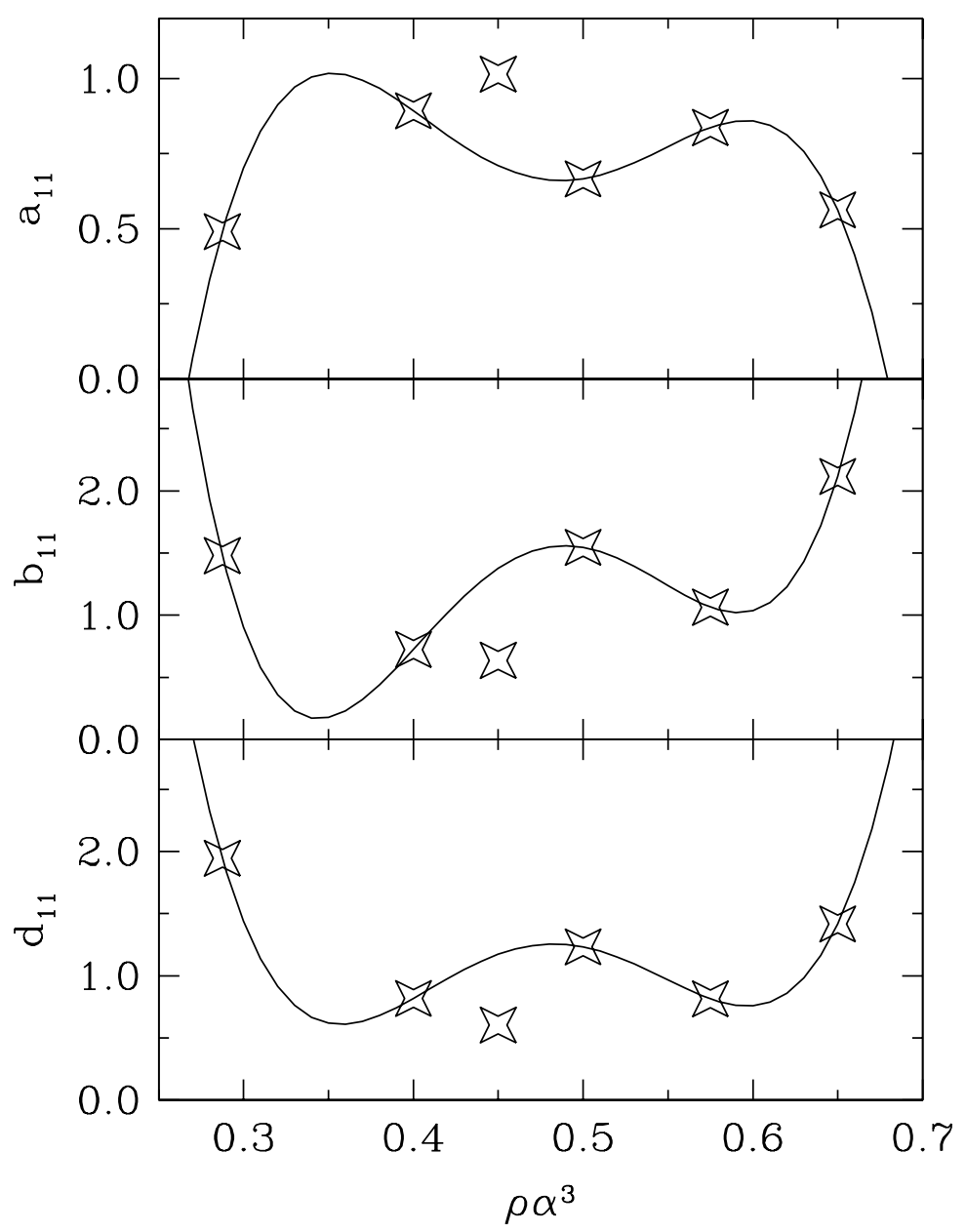

FIG. 5: R. Fantoni and G. Pastore 


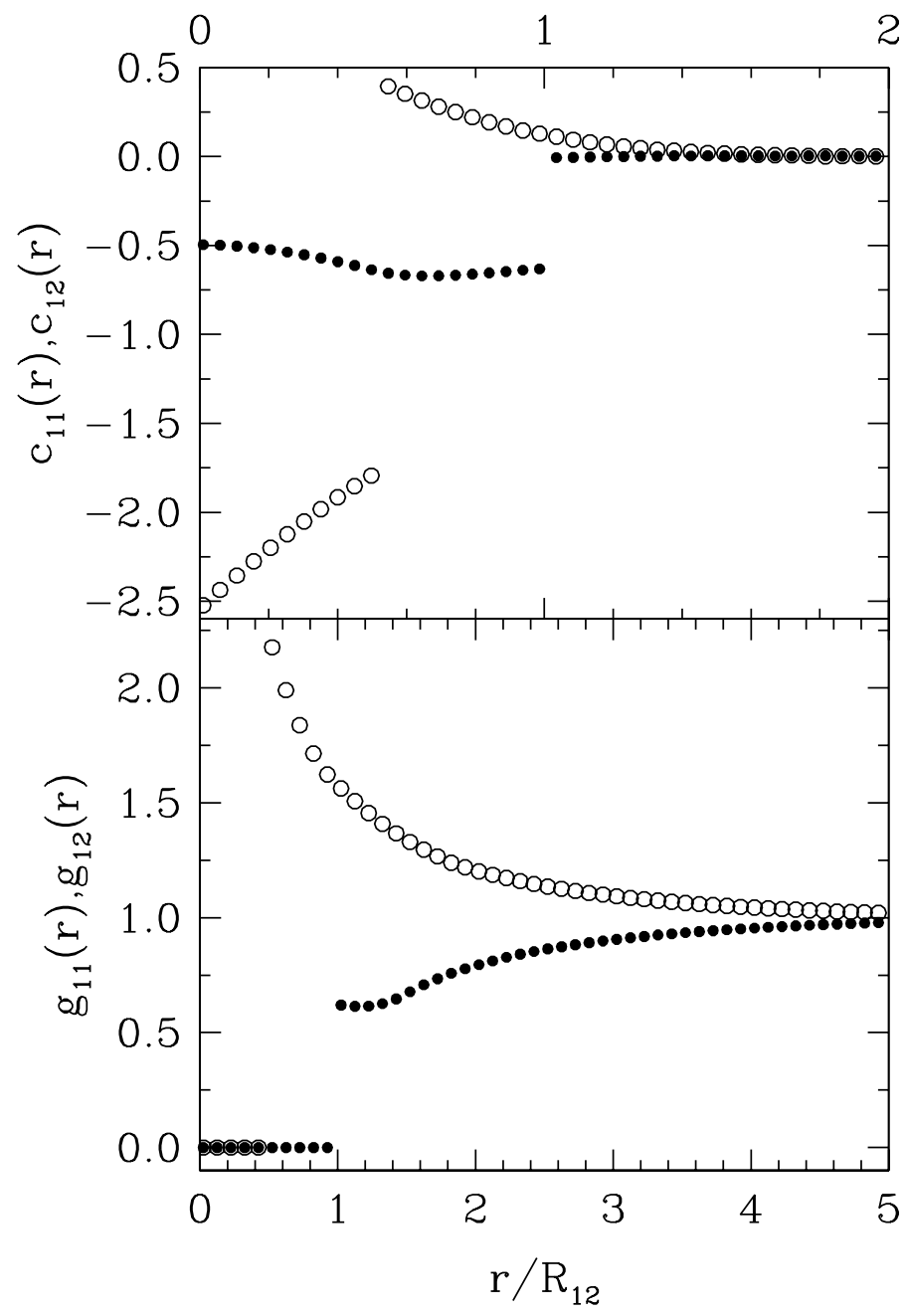

FIG. 6: R. Fantoni and G. Pastore 


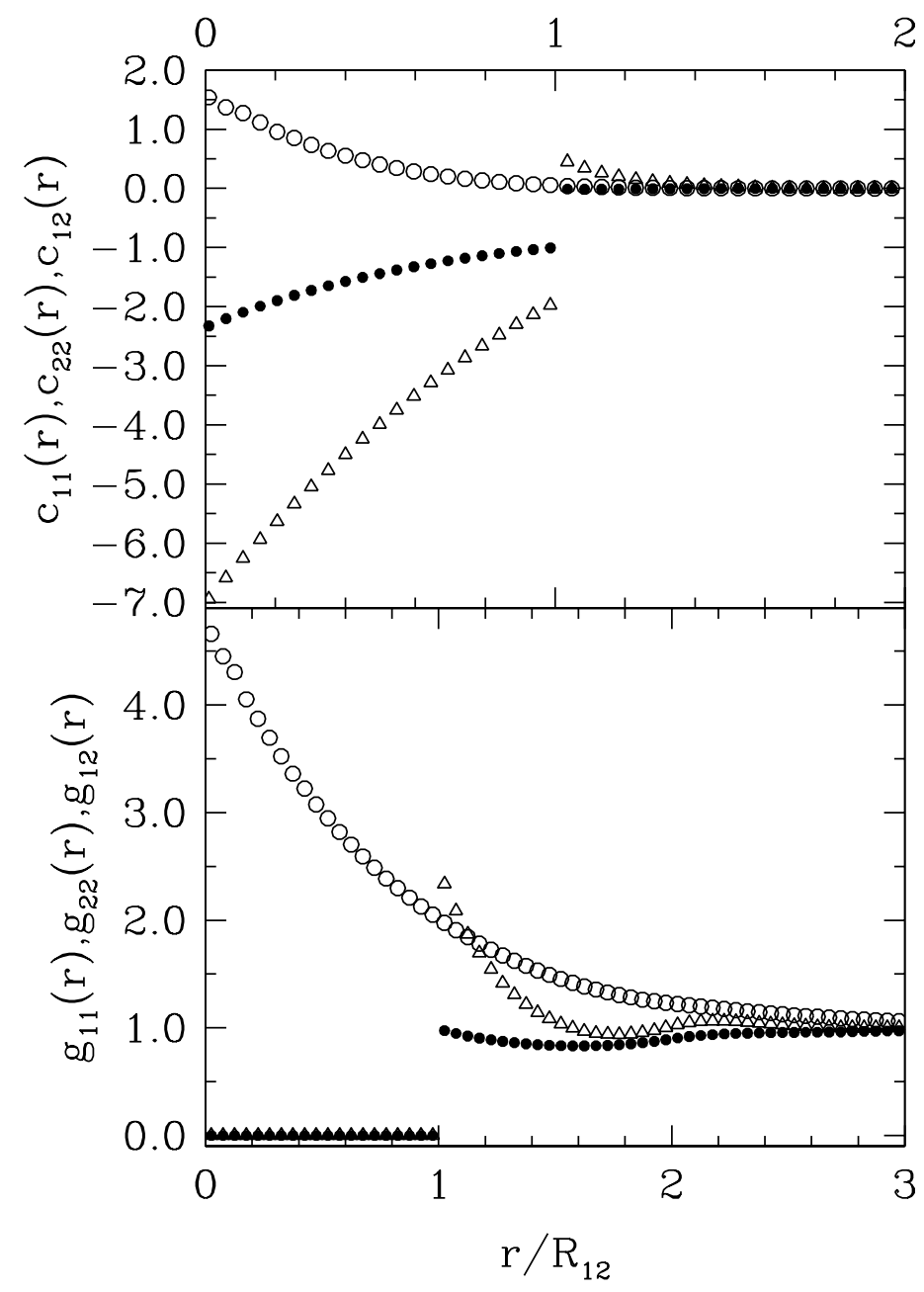

FIG. 7: R. Fantoni and G. Pastore 